\title{
Acknowledgement to Reviewers of Molbank in 2015
}

\author{
Molbank Editorial Office \\ Published: 25 January 2016 \\ MDPI AG, Klybeckstrasse 64, CH-4057 Basel, Switzerland; molbank@mdpi.com
}

The editors of Molbank would like to express their sincere gratitude to the following reviewers for assessing manuscripts in 2015.

We greatly appreciate the contribution of expert reviewers, which is crucial to the journal's editorial decision-making process. Several steps have been taken in 2015 to thank and acknowledge reviewers. Good, timely reviews are rewarded with a discount off their next MDPI publication. By creating an account on the submission system, reviewers can access details of their past reviews, see the comments of other reviewers, and download a letter of acknowledgement for their records. This is all done, of course, within the constraints of reviewer confidentiality. Feedback from reviewers shows that most see their task as a voluntary and mostly unseen work in service to the scientific community. We are grateful to our reviewers for the contribution they make.

Adler, Marc J.

Aitken, R. Alan

Alajarín, Mateo

Benedec, Daniela

Besson, Thierry

Bouquillon, Sandrine

Caselli, Alessandro

Castñeiras, Alfonso

Chassaing, Stephan

Chevallier, Floris

Cîrcu, Viorel

Claramunt, Rosa M.

Clark, Daniel A.

de la Hoz, Antonio

De Luca, Lidia

Detert, Heiner

Dias, Luiza Rosaria Sousa

Díaz, David

Ducki, Sylivie

Fañanás-Mastral, Martín

Feng, Yu

Ferraz, Ricardo

Francioso, Antonio

Gaboriaud, Nicolas

Gan, Quan

Gentilucci, Luca

Gimnez, Raquel

Giustiniano, Mariateresa

González-Muñiz, Rosario

Gourand, F.

Kaczor, Agnieszka
Kayaki, Yoshihito
Kitamura, Mitsuru
Kitano, Yoshikazu
Kotora, Martin
Kozlowski, Henryk
Larionov, Oleg V.
Lee, Kwang-Ming
Lee, Hon Man
Leoni, Alberto
Li, Rui
Li, Yanmei
Lindhardt, Anders T.
Luconi, Lapo
Lukas, Thomas J.
Maas, Gerhard
Mandal, Debashis
Maslov, Mikhail A.
McGlinchey, Michael J.
Melguizo-Guijarro, Manuel
Mino, Takashi
La Motta, Concettina
Murafuji, Toshihiro
Murata, Toshihiro
Musiol, Robert
Okamoto, Noriko
Opletalova, Veronika
Ozeki, Yasuhiro
Penoni, Andrea
Pérez, Dolores

Robledo, Sara M.

Ryu, Shi Yong

Salvio, Riccardo

Samoshin, Vyacheslav V.

Schirrmacher, Ralf

Seifert, Tina

She, Zhi-Gang

Shin, Dongyun

Sikva, Artur M.S.

Snyder, James P.

Stephen, Michael Rajesh

Sucunza, David

Sung, Wang Chou

Svete, Jurij

Sydnes, Leiv Kr.

Takasu, Kiyosei

Talhi, Oualid

Thomas, Eric

Thompson, Sam

Torroba, Tomas

Tsukamoto, Masaki

Tumkevičius, Sigitas

Urabe, Hirokazu

Van Aerschot, Arthur

Vanderzande, Dirk J. M.

Vanelle, Patrice

Wang, Lan-Ying

Wang, Zhiqiang

Yamamura, Masaki

Yang, Renqiang 
Gribble, Gordon W.

Guo, Min

Hadjikakou, Sotiris K.

Hadjipavlou-Litina, Dimitra

Heimgartner, Heinz

Hinkle, Robert J.

Hirano, Koji

Hoskovcova, Irena

$\mathrm{Hu}$, Chun

Huang, Jiann-Jyh
Pérez, Andy J.

Pescitelli, Gennaro

Podlech, Joachim

Pujol, Maria Dolors

Qiu, Ming-Hua

Quadrelli, Paolo

Quiroga, Jairo

Rajca, Andrzej

Rayat, Sundeep

Rinner, Uwe
Yokomatsu, Tsutomu

Yu, Chin-Yang

Zakrzewski, Jerzy

Zeng, Huaqiang

Zeng, Xiaoming

Zeng, Dexing

Zhai, Xiang

Zhu, Jianglong

Zi, Guofu

(C) 2016 by the authors; licensee MDPI, Basel, Switzerland. This article is an open access article distributed under the terms and conditions of the Creative Commons by Attribution (CC-BY) license (http://creativecommons.org/licenses/by/4.0/). 